\title{
Fatigue Damage Detection and Risk Assessment via Wavelet Transform and Neural Network Analysis of Ultrasonic Signals
}

\author{
HASSAN ALQAHTANI ${ }^{1}$
}

${ }^{1}$ Taibah University

October 1, 2021

\begin{abstract}
This paper develops a data-driven autonomous method for detection of fatigue damage and classification of the associated damage risk in mechanical structures, based on ultrasonic signal energy. The underlying concept is built upon attenuation of the signal and stability of the attenuation process. The attenuation provides pertinent information for damage quantification, whereas the stability represents resistance towards the fatigue damage growth. The proposed neural network (NN) model has been trained using the scaled conjugate-gradient back-propagation method. The NN model is capable of damage detection and damage classification into five classes of increasing risk. The Daubechies wavelet transform has been used to reduce the noisy pattern of the ultrasonic signal energy by using the associated approximation coefficients. The results show that the proposed method of approximation signal energy can detect and classify the damage with an accuracy of up to $98.5 \%$.
\end{abstract}

\section{Hosted file}

Fatigue Damage Detection and Risk Assessment via Wavelet Transform and Neural Network Analysis of Ultr available at https://authorea.com/users/438987/articles/540029-fatigue-damage-detection-andrisk-assessment-via-wavelet-transform-and-neural-network-analysis-of-ultrasonic-signals

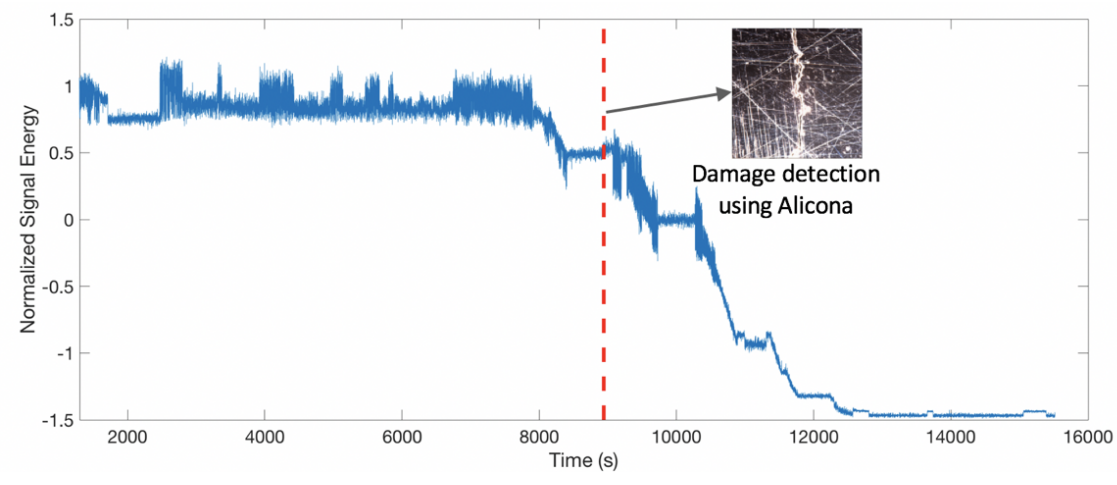



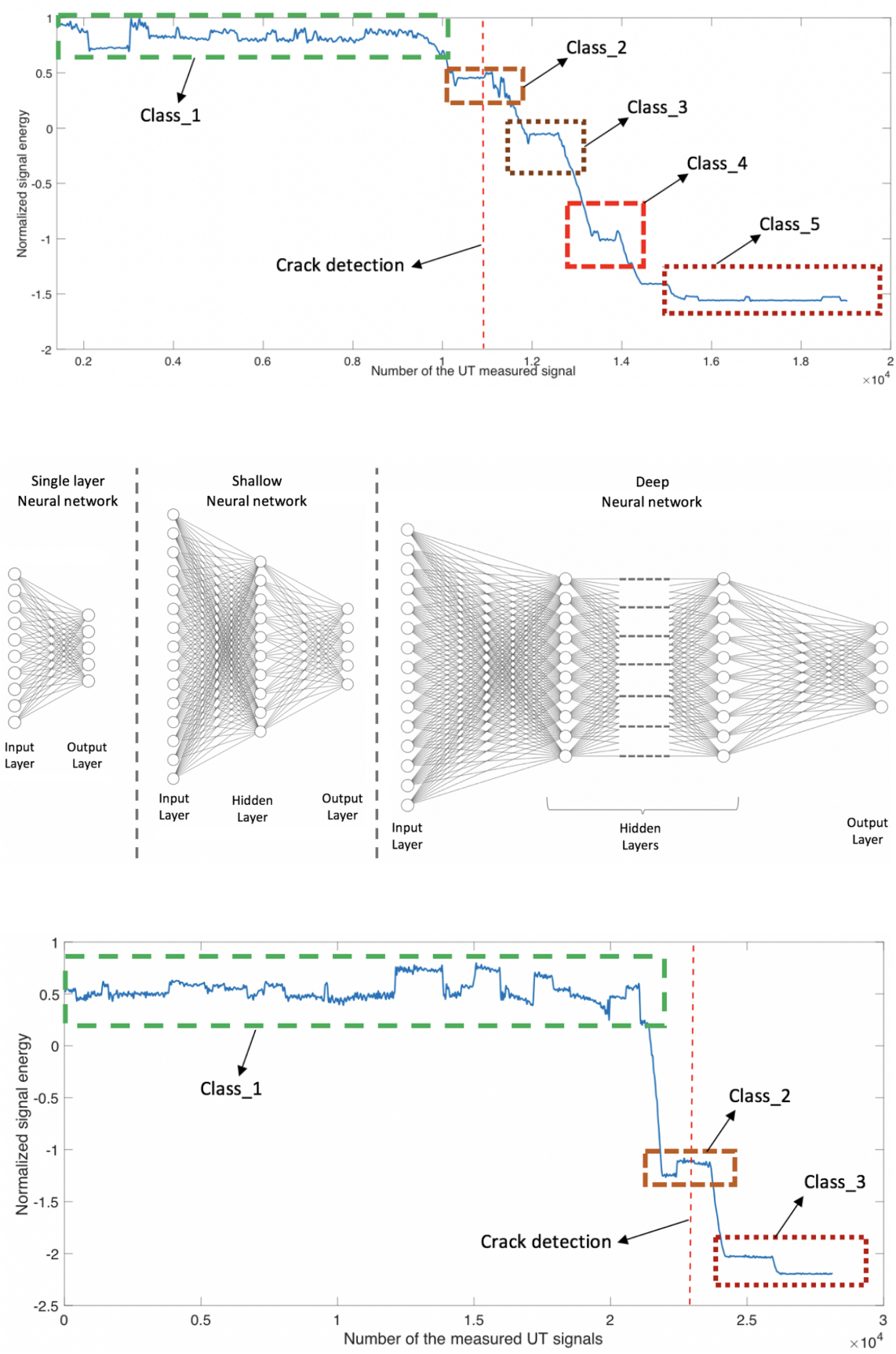

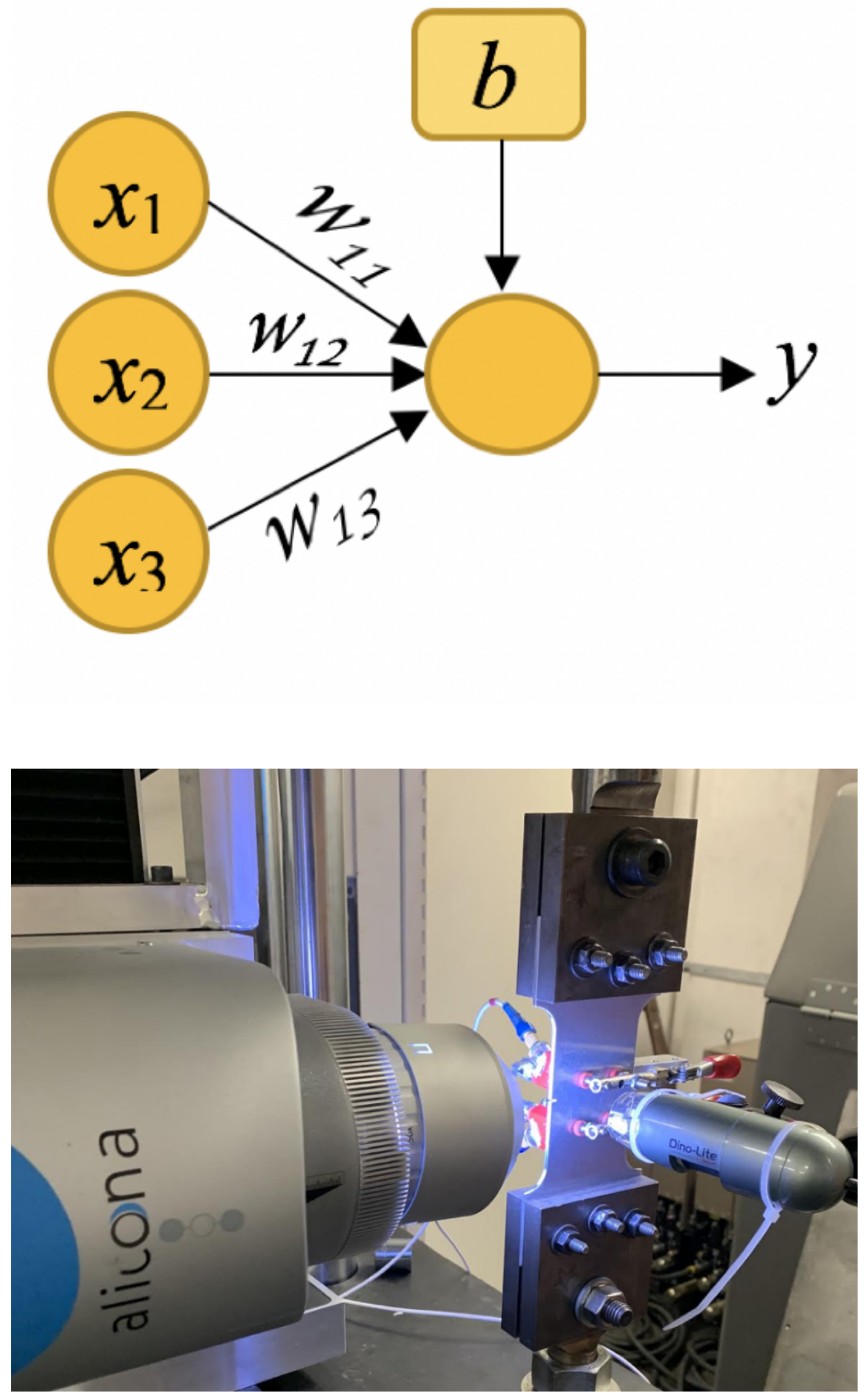

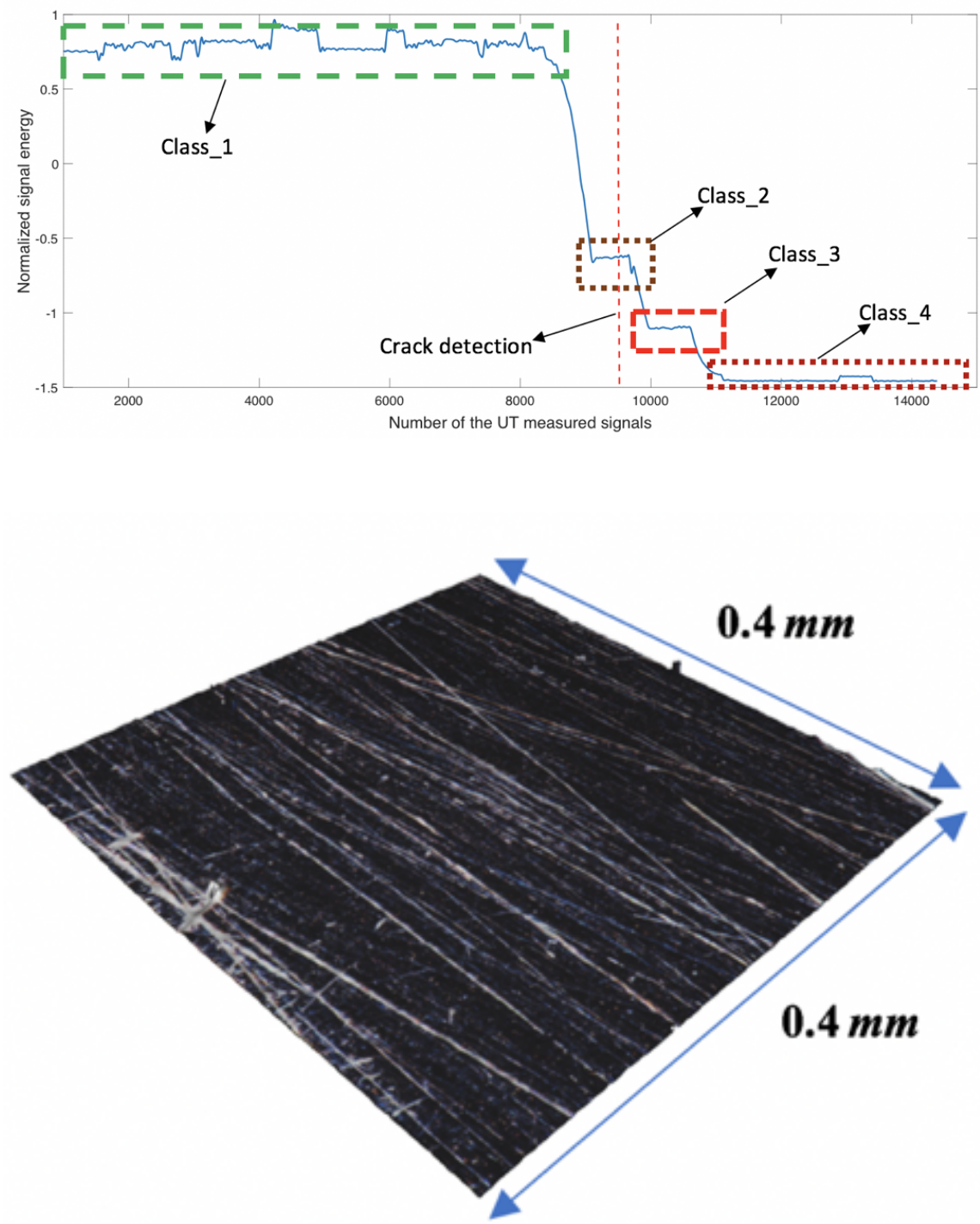

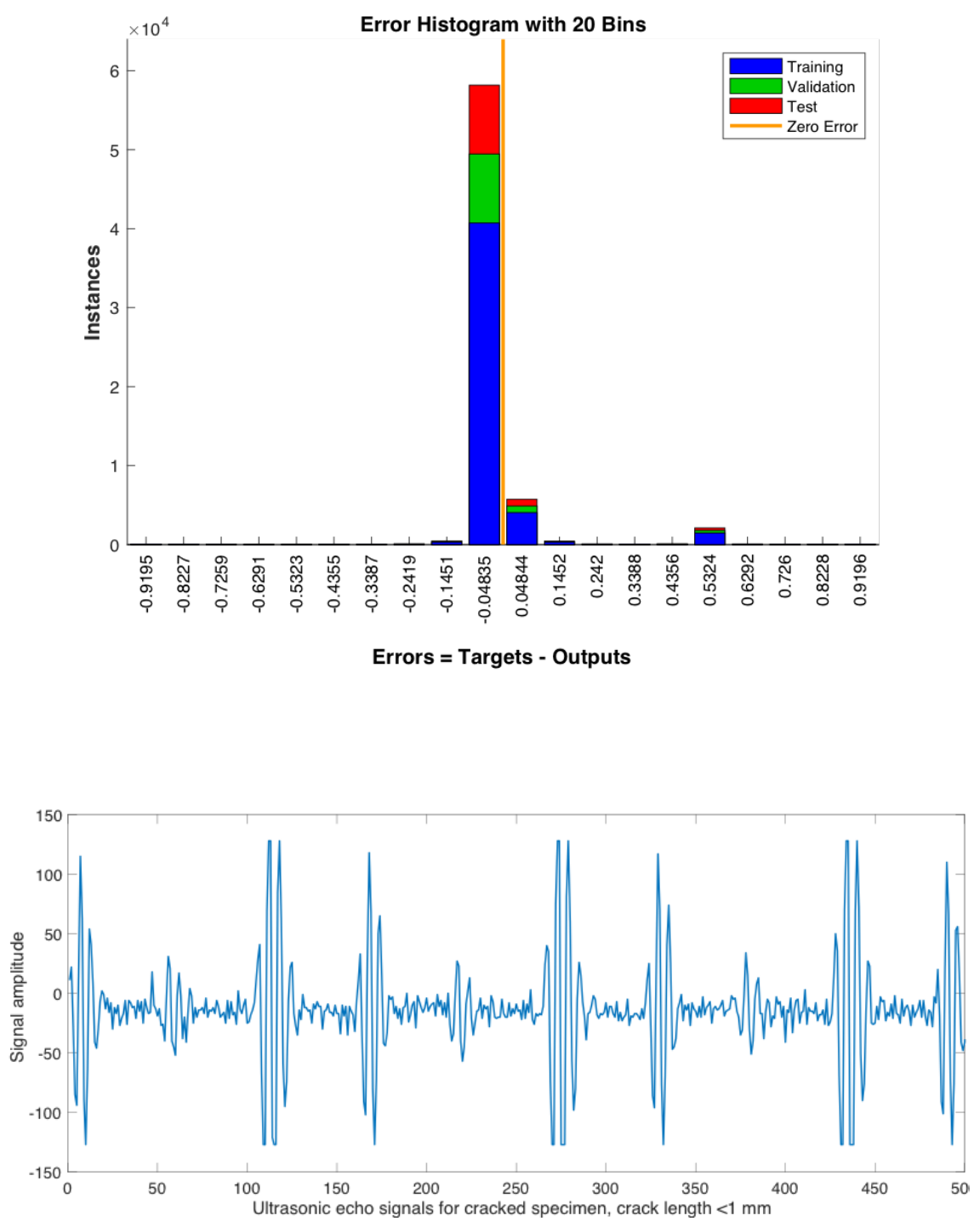

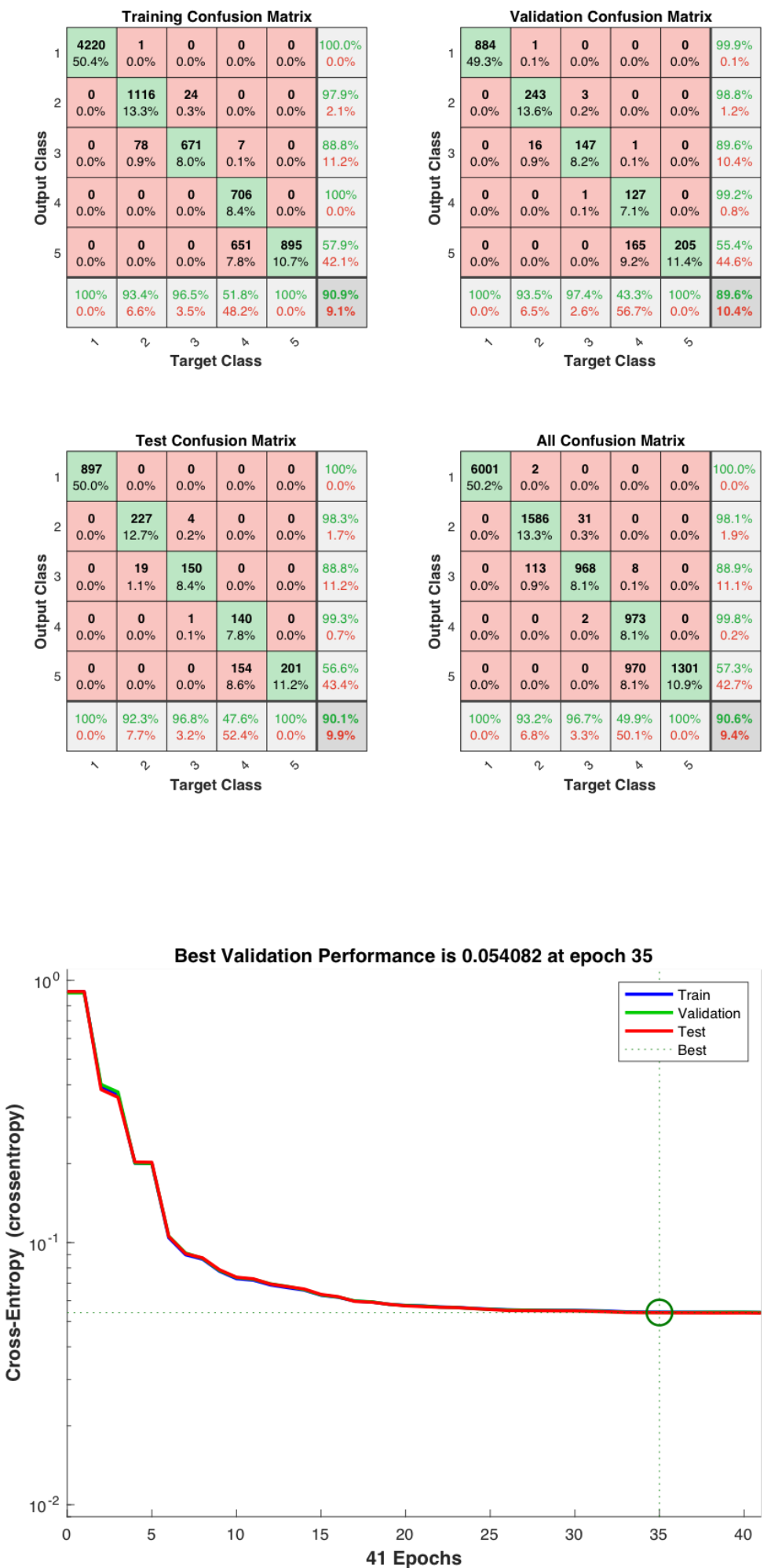

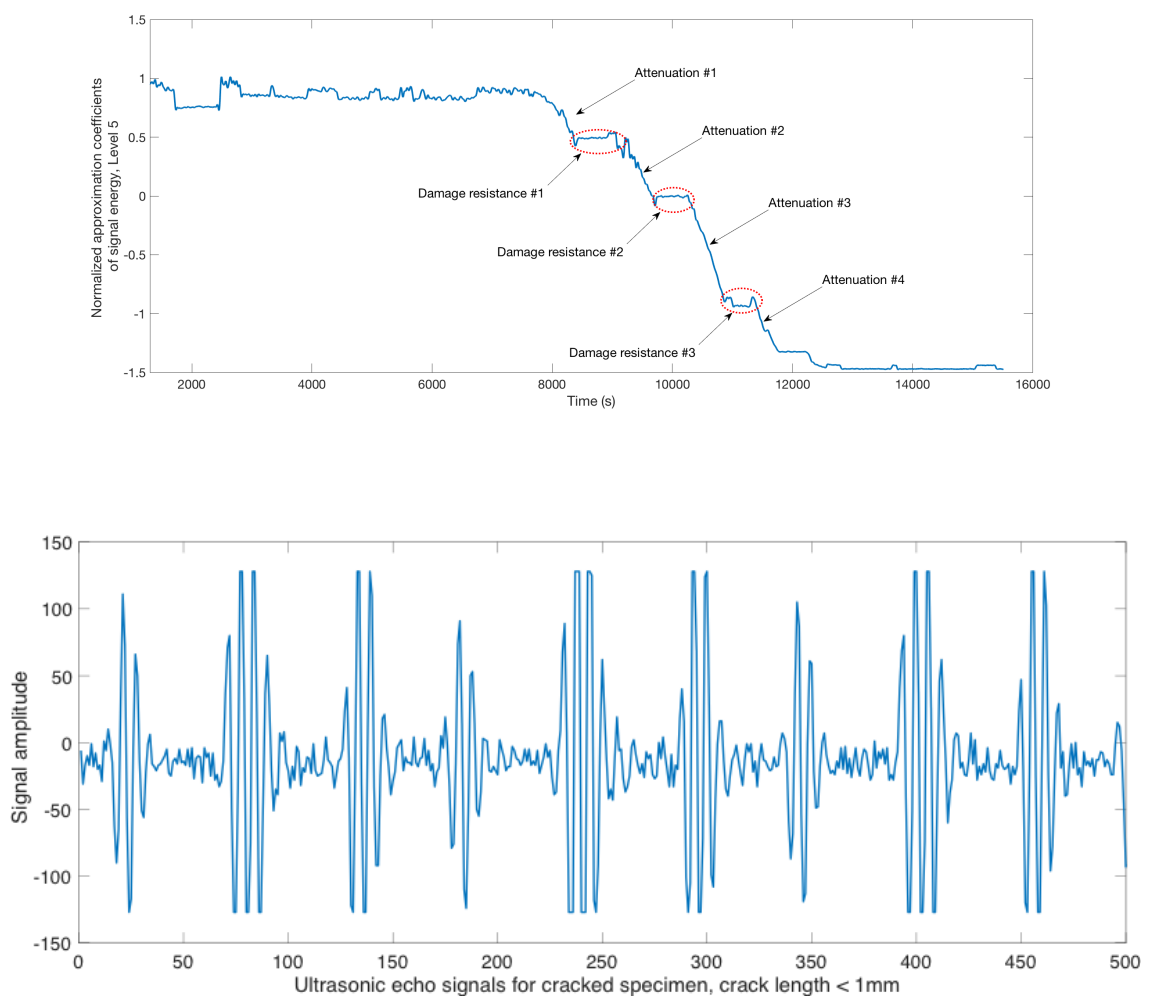

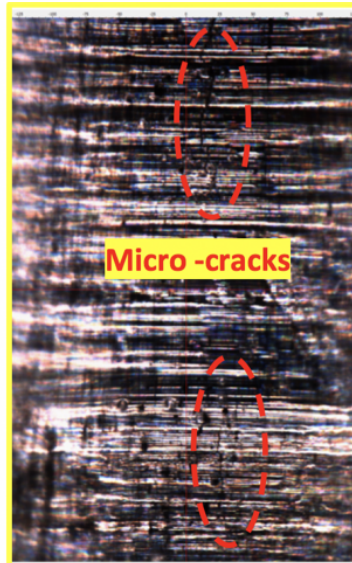

Damage_class_1

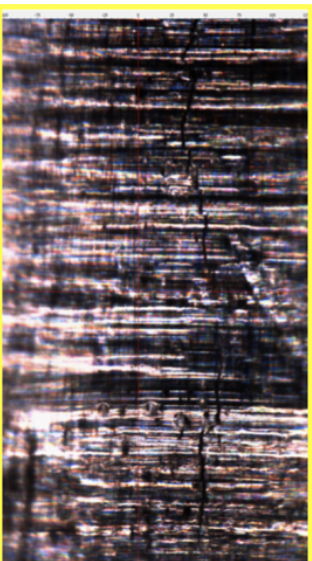

Damage_class_2
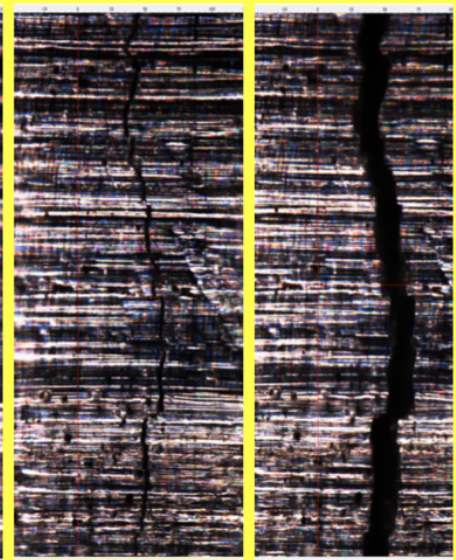

Damage_class_3 Damage_class_4 

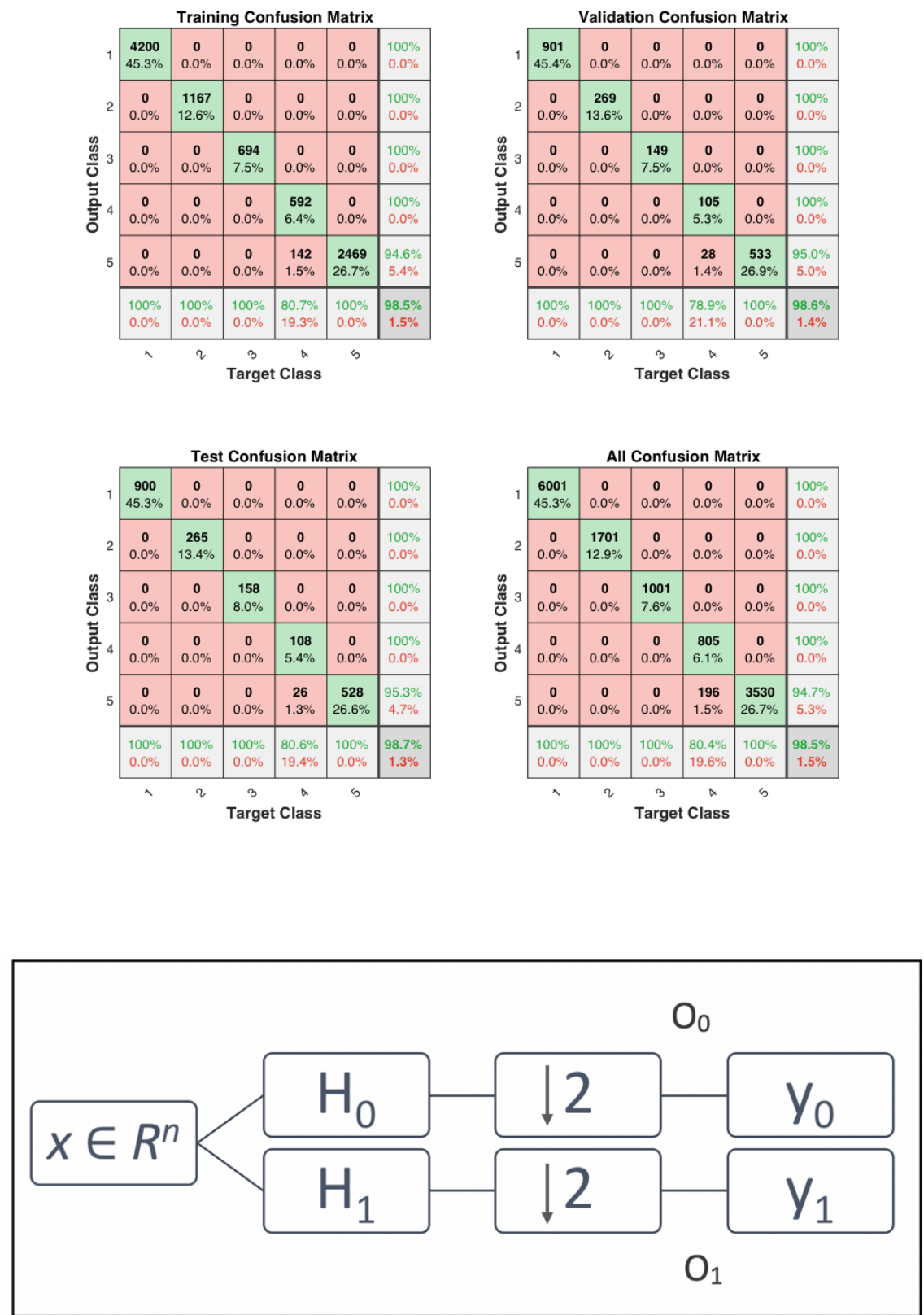

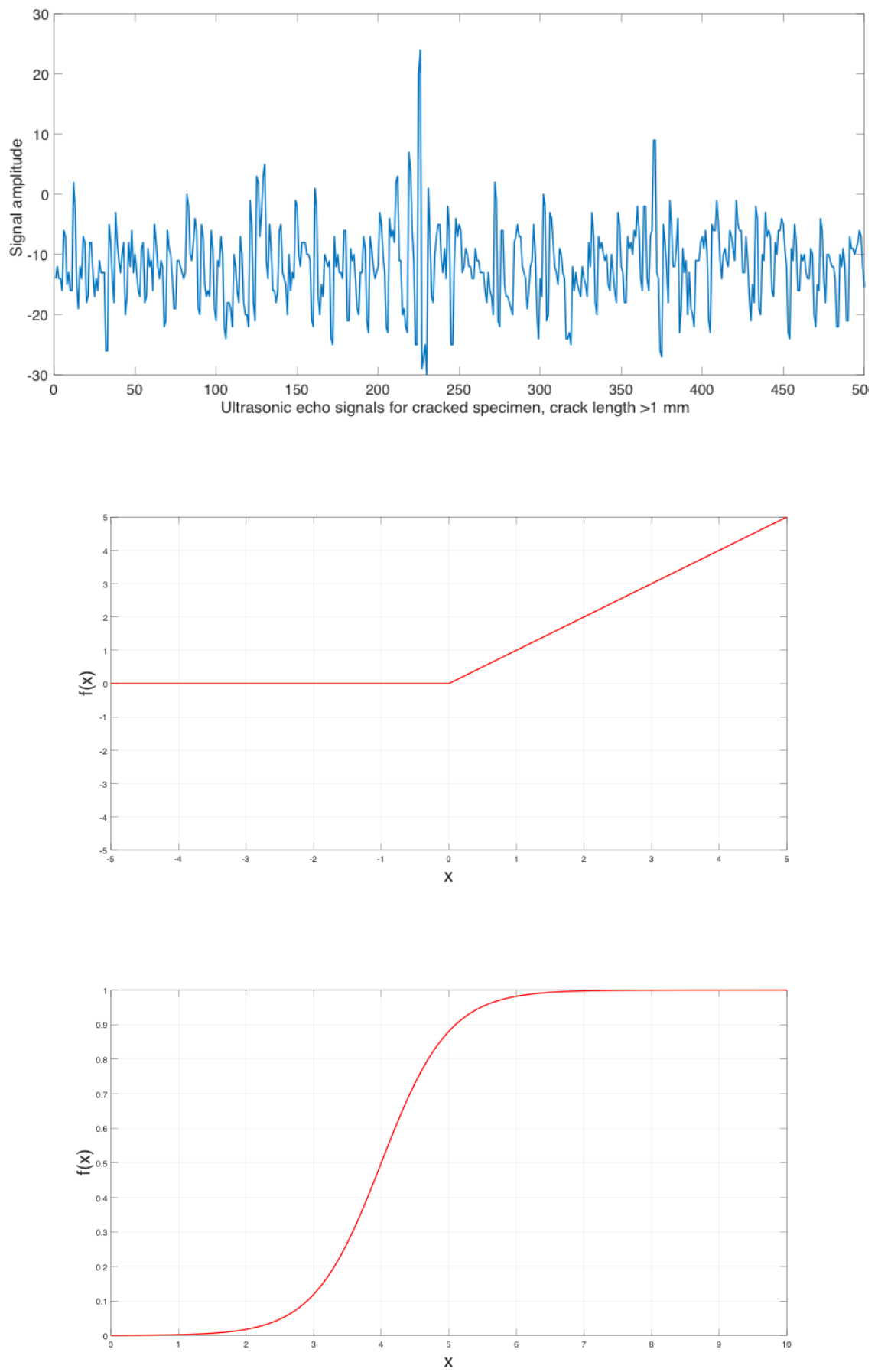

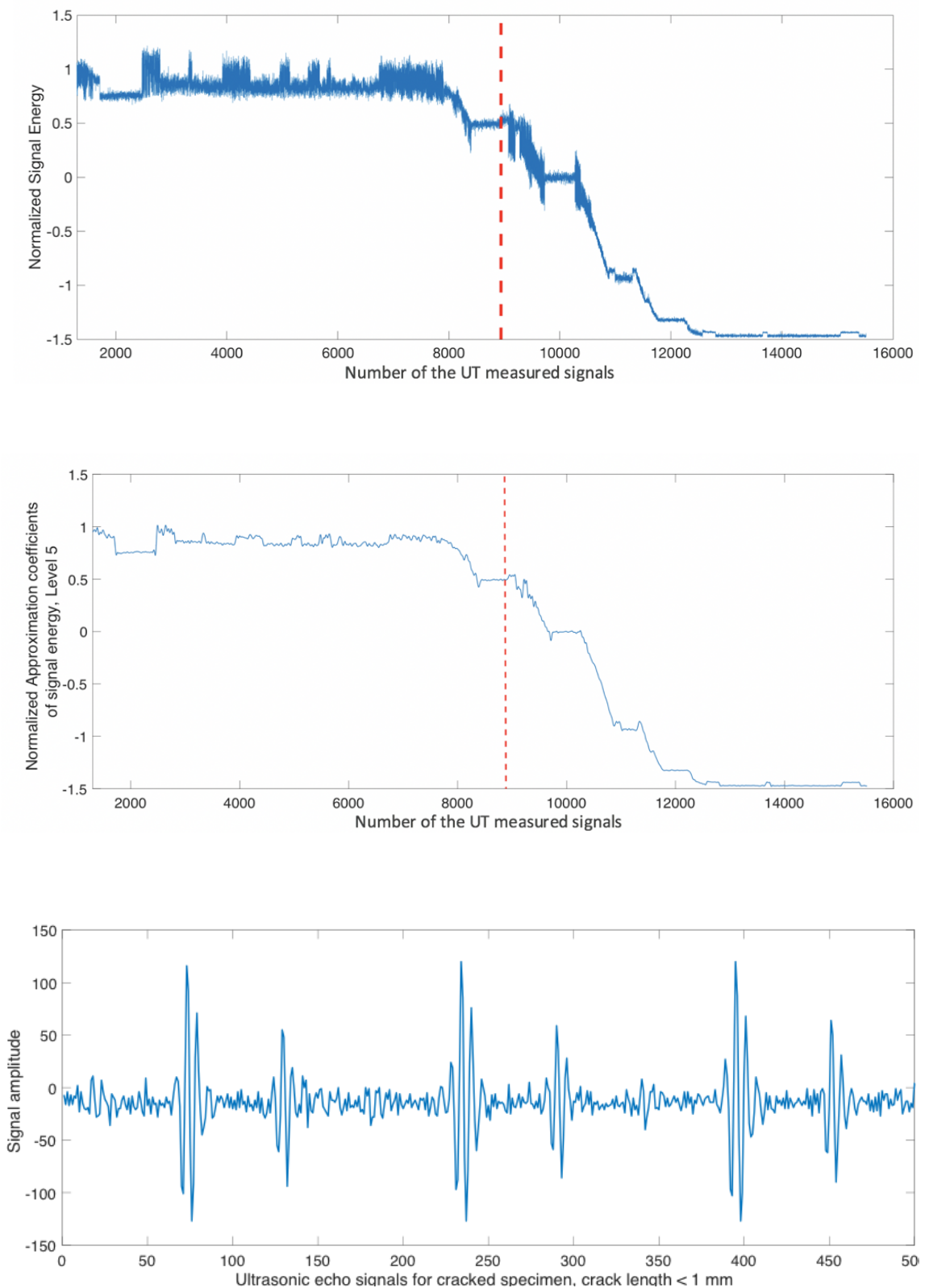
- Initialize random weights.

- Calculate the activation rate of hidden layers from the input data and assigned weights between input layer and hidden layers.

- Repeat step 2 for next layers until the output layer, where the input data of each layer is the outputs of the previous layer, and the weights are the assigned weights between the previous layer and current layer.

- Find the error between outputs of ANN and the desired outputs.

- Adjust the weights between output layer and hidden layer using the current weights and calculated error.

- Adjust the weights between hidden layer and input layer.

- Repeat step 2 to step 6 for all training data.

- Repeat step 2 to step 7 till the error converges to an acceptable limit.

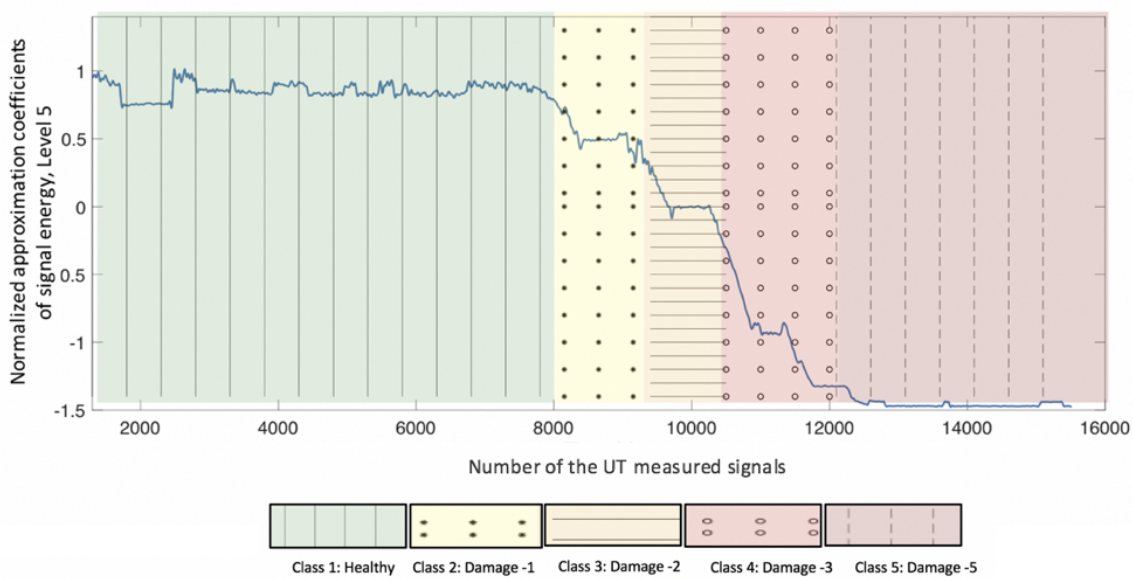



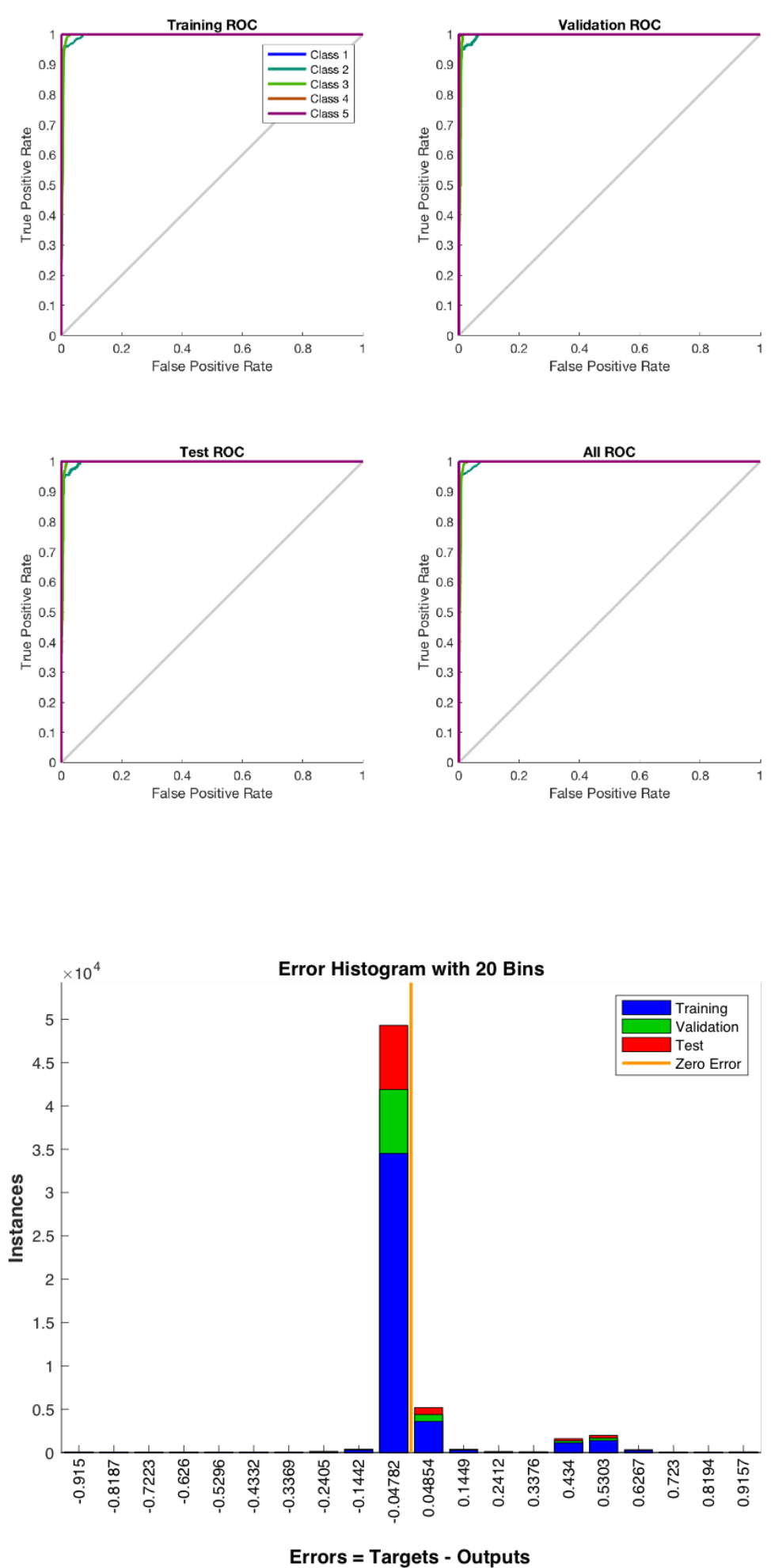

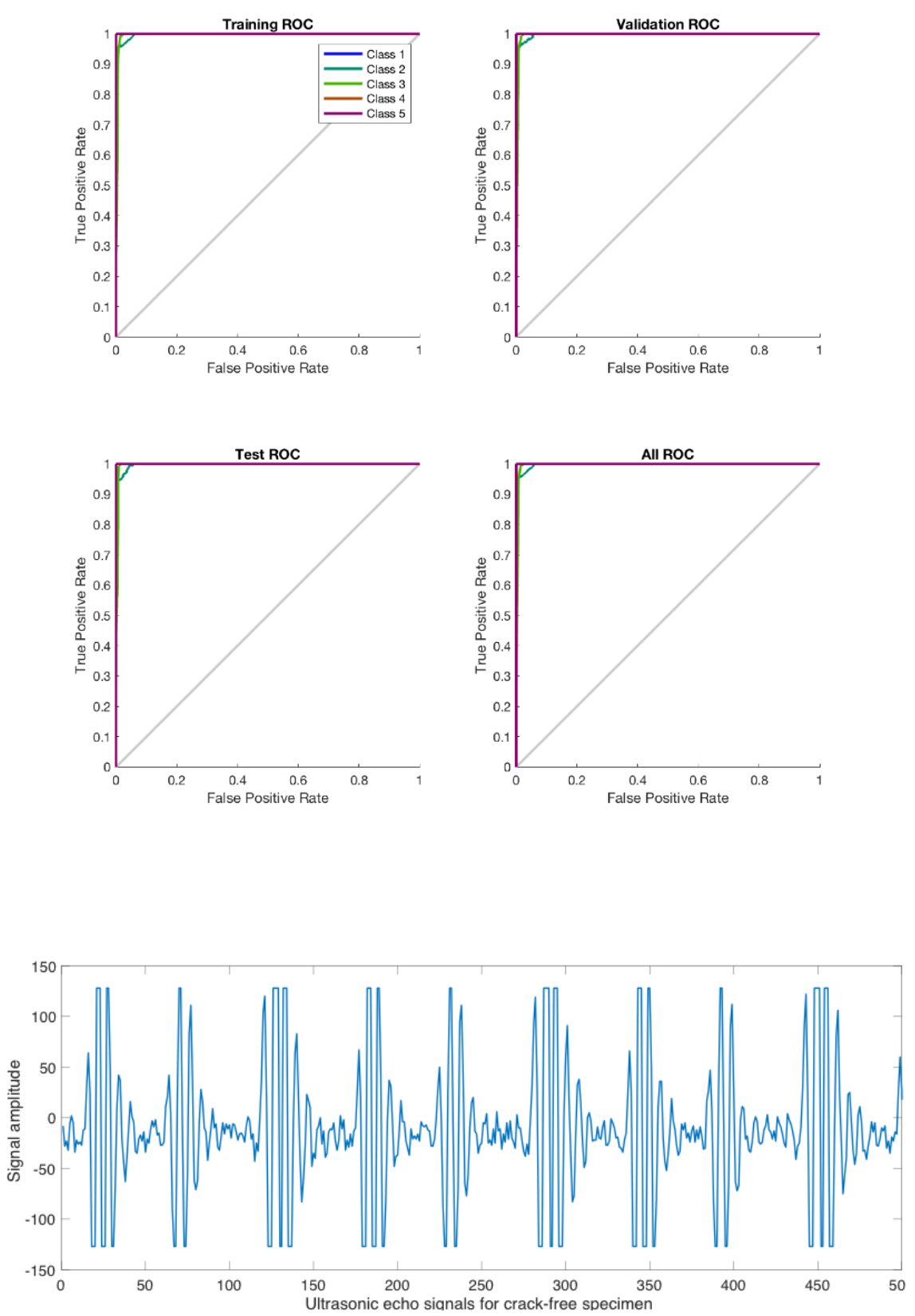


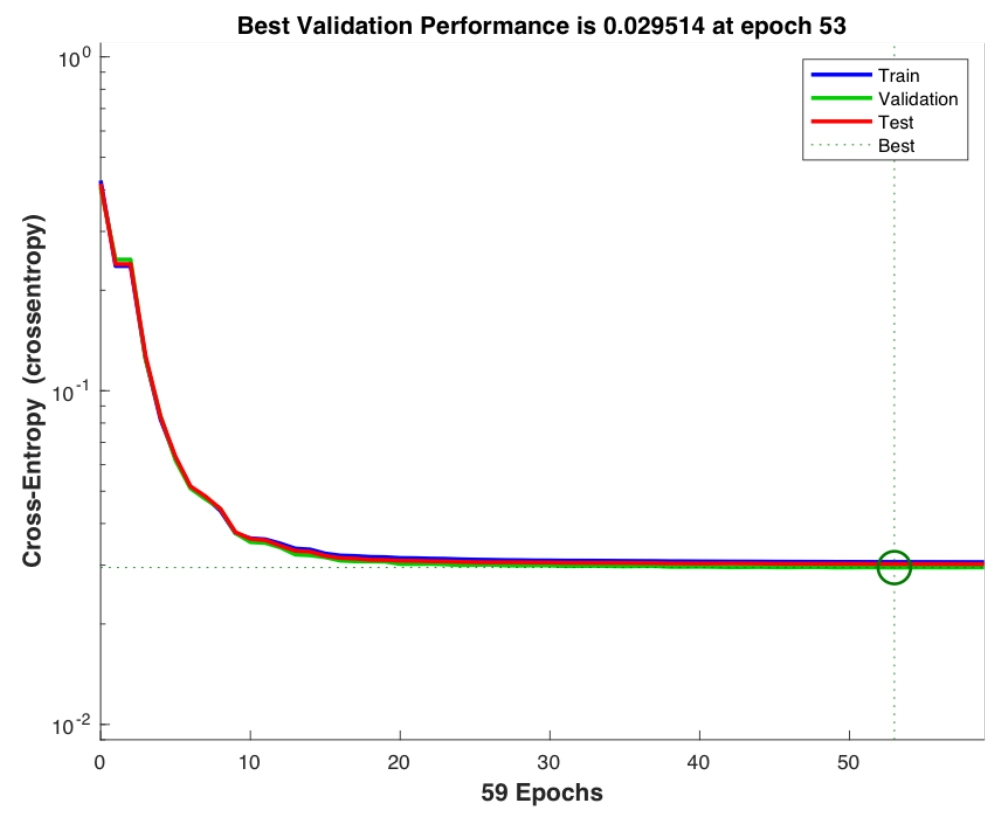

